CrossMark \& click for updates

Cite this: Phys. Chem. Chem. Phys., 2016, 18, 14129

Received 13th January 2016, Accepted 3rd May 2016

DOI: $10.1039 / \mathrm{c} 6 \mathrm{cp} 00260 \mathrm{a}$

www.rsc.org/pccp

\section{Investigation of the potassium fluoride post deposition treatment on the CIGSe/CdS interface using hard X-ray photoemission spectroscopy - a comparative study}

\author{
Bünyamin Ümsür, ${ }^{\text {ab }}$ Wolfram Calvet, ${ }^{a}$ Alexander Steigert, ${ }^{a}$ Iver Lauermann, ${ }^{a}$ \\ Mihaela Gorgoi, ${ }^{a}$ Karsten Prietzel, ${ }^{a}$ Dieter Greiner, ${ }^{a}$ Christian A. Kaufmann, ${ }^{a}$ \\ Thomas Unold ${ }^{a}$ and Martha Ch. Lux-Steiner ${ }^{\mathrm{ab}}$
}

\begin{abstract}
The impact of the potassium fluoride post deposition treatment on CIGSe chalcopyrite absorbers is investigated by means of depth resolved hard X-ray photoemission spectroscopy of the near surface region. Two similar, slightly $\mathrm{Cu}$-poor $\mathrm{CIGSe}$ absorbers were used with one being treated by potassium fluoride prior to the chemical bath deposition of an ultrathin CdS layer. The thickness of the CdS layer was chosen to be in the range of about $10 \mathrm{~nm}$ in order to allow the investigation of the CIGSe/CdS interface by the application of hard X-rays, increasing the information depth up to $30 \mathrm{~nm}$. Besides strong intermixing on both samples, an increased $\mathrm{Cu}$ depletion of the KF treated absorber was observed in combination with an increased accumulation of $\mathrm{Cd}$ and $\mathrm{S}$. In addition, a general shift of about $0.15 \mathrm{eV}$ to higher binding energies of the CIGSe valence band at the absorber surface as well as the CIGSe and CdS related core levels was measured on the KF treated sample. This phenomenon is attributed to the impact of additional cadmium which acts as donor and releases further electrons into the conduction band of the absorber. Finally, the electrons accumulate at the CdS surface after having passed the interface region. This additional surface charge leads to a pronounced shift in the photoemission spectra as observed on the KF treated CIGSe absorber compared to the non-treated absorber.
\end{abstract}

\section{Introduction}

Thin film solar cells based on polycrystalline $\mathrm{Cu}(\mathrm{In}, \mathrm{Ga}) \mathrm{Se}_{2}$ (CIGSe) chalcopyrite absorbers already have reached efficiencies exceeding those of polycrystalline silicon solar cells on the laboratory scale. ${ }^{1}$ It is well-known that alkaline metals such as sodium are crucial for the performance of CIGSe solar cells although their impact on device preparation is not yet fully understood. The mechanism of sodium incorporation into the growing absorber film has been intensively discussed on a theoretical and on an experimental basis in terms of absorber growth, interdiffusion mechanisms and device quality. ${ }^{2-6}$ By using a combined NaF and KF post deposition treatment (PDT) of a CIGSe absorber grown on polyimide foil Chirilă et $a .^{7}$ were able to prepare a world record device at that time with a cell efficiency of $20.4 \%$. They found a remarkable modification of the CIGSe surface after applying the KF-PDT. With surface

\footnotetext{
${ }^{a}$ Helmholtz-Zentrum-Berlin, Hahn-Meitner-Platz 1, D-14109 Berlin, Germany. E-mail: buenyamin.uemsuer@helmholtz-berlin.de

${ }^{b}$ Freie Universität Berlin, Department of Physics, Arnimallee 14, D-14195 Berlin, Germany
}

sensitive X-ray photoelectron spectroscopy (XPS) they observed a much stronger $\mathrm{Cu}$ and $\mathrm{Ga}$ depletion of the surface region compared to the untreated absorber. They concluded that the increased deficiency of $\mathrm{Cu}$ at the CIGSe surface leads to an enhanced Cd diffusion into the absorber during the chemical bath deposition (CBD) of the CdS buffer layer even at low process temperatures. This phenomenon is also supported by theoretical studies conducted by Kiss et al. ${ }^{8,9}$ Recently, we also demonstrated with hard X-ray photoelectron spectroscopy (HAXPES) that the amount of Cd diffusing into the interface region of the absorber during the CBD of CdS is dependent on the $\mathrm{Cu}$ concentration. ${ }^{10}$ The penetration of Cd into the CIGSe absorber becomes even more pronounced upon annealing in ultra-high vacuum (UHV) at $350{ }^{\circ} \mathrm{C}$ indicating that the $\mathrm{Cd}$ diffusion in CIGSe might be correlated with the $\mathrm{Cu}$ migration in this material which is directly connected to the formation of $\mathrm{Cu}$ vacancies $\left(\mathrm{V}_{\mathrm{Cu}}\right) \cdot{ }^{11}$

In the present work, we examine the changes of the CIGSe/ CdS interface induced by a KF-PDT of the CIGSe absorber. For this purpose, two standard CIGSe samples (slightly Cu-poor) were grown in the same batch in the CIGSe deposition tool of the HZB base line ${ }^{12}$ with one sample kept in its as-grown state, 
while the other one underwent a KF-PDT in a separate process step. Afterwards, ultrathin CdS layers were deposited by CBD on both absorbers under the same conditions with an estimated thickness of $10 \mathrm{~nm}$, which allows the simultaneous measurement of both, the CIGSe absorber and the CdS buffer layer as well as the corresponding interface region using HAXPES at different excitation energies. The aim was to investigate the interface region especially focussing on $\mathrm{Cu}$ and $\mathrm{Cd}$ species, which are believed to play an important role in the formation of the CIGSe/CdS interface.

\section{Experimental details}

\subsection{Sample preparation}

Polycrystalline CIGSe thin films were prepared on molybdenum coated soda lime glass (SLG) substrates using a modified threestage coevaporation process. Details on this process are given in ref. 13. The $\mathrm{Cu}$-content of the completed CIGSe film is mostly determined by the duration of the second and the third stage after and before the stoichiometric point of the compound material is reached as measured with optical real-time methods. The evaporation of In, Ga, and Se beyond this point results in a $\mathrm{Cu}$-poor CIGSe absorber. By shortening the third stage accordingly, Cu-rich absorbers can be also produced. For the experiment at hand, two Cu-poor CIGSe samples were grown on $5 \mathrm{~cm} \times 5 \mathrm{~cm}$ large SLG substrates with bulk compositions of $[\mathrm{Cu}] /([\mathrm{In}]+[\mathrm{Ga}])=0.88$ and $[\mathrm{Ga}] /([\mathrm{In}]+[\mathrm{Ga}])=0.32$, as determined by X-ray fluorescence. Directly after finishing the absorber preparation process, one sample was stored in nitrogen atmosphere in order to minimize the impact of air exposure whereas on the other sample a KF-PDT was applied. The post deposition treatment was performed ex situ in a separate UHV evaporation chamber for $20 \mathrm{~min}$ at a substrate temperature of $330{ }^{\circ} \mathrm{C}$ in the presence of $\mathrm{KF}$ and Se molecular beams. Afterwards, thin CdS layers were deposited by CBD using $0.0189 \mathrm{M}$ cadmium acetate dihydrate $\left(\mathrm{Cd}\left(\mathrm{C}_{2} \mathrm{H}_{3} \mathrm{O}_{2}\right)_{2} \cdot 2 \mathrm{H}_{2} \mathrm{O}\right)$ in $11.25 \mathrm{ml}$ aqueous $\mathrm{NH}_{3}(25 \%)$ and $0.9565 \mathrm{M}$ thiourea $\left(\mathrm{H}_{2} \mathrm{NCSNH}_{2}\right)$ in $100 \mathrm{ml}$ water which were mixed together and filled up by distilled water to a total volume of $150 \mathrm{ml}$. Then, both samples were simultaneously dipped into the chemical bath for $50 \mathrm{~s}$ at $60{ }^{\circ} \mathrm{C}$ leading to $10 \mathrm{~nm}$ thick CdS layers as estimated from the standard CdS deposition process. Afterwards, they were rinsed with deionized water and dried in an ultrapure nitrogen gas stream. Prior to the wet chemical CdS deposition process, both samples were cut into small pieces of $4 \mathrm{~mm} \times 8 \mathrm{~mm}$ size in order to be able to mount them both on one sample holder. The CIGSe/CdS and KF-PDT CIGSe/CdS samples were then rapidly transferred to the UHV based analysis system dedicated for HAXPES using a small transport box flooded with nitrogen.

\subsection{Sample characterisation}

The HAXPES measurements were performed at the HIKE end station ${ }^{14}$ which is operated at the KMC-1 beamline ${ }^{15}$ at the BESSY II synchrotron facility in Berlin providing variable photon energies up to $12 \mathrm{keV}$. For the photoemission experiments, which are presented in this paper, excitation energies of 2030, 3000, 4000, 5000, and $6000 \mathrm{eV}$ were used, yielding variable information depths which are calculated for the Cd3d emission as $9 \mathrm{~nm}, 13 \mathrm{~nm}, 17 \mathrm{~nm}, 20 \mathrm{~nm}$, and $24 \mathrm{~nm}$, respectively. The analyser at the HIKE end station consists of a R4000 hemispherical photoelectron spectrometer manufactured by Scienta Gammadata optimized for high kinetic energy. All spectra in this work were recorded at a constant pass energy of $200 \mathrm{eV}$. The X-ray beam was horizontally polarized and hit the sample in grazing incidence geometry under an angle of $3^{\circ}$ towards the surface. The generated photoelectrons were detected in the polarization plane perpendicular to the beam while the incident beam intensity was monitored with an $\mathrm{N}_{2}$ ionization chamber and kept constant for all measurements (top-up mode of the storage ring with $298 \mathrm{~mA}$ ring current). The spot size of the beam on the sample under grazing incidence conditions was in the range of $0.1 \mathrm{~mm}$ in height and $1 \mathrm{~mm}$ in length, reducing the influence of surface roughness and inhomogeneity of the CdS film on the accuracy of the measurements due to averaging effects. The entrance slit of the electron analyser with $0.5 \mathrm{~mm}$ width was kept constant for all measurements. In addition, the samples were imaged with a Zeiss Leo 1530 scanning electron microscope (SEM) after the HAXPES measurements were done.

\section{Results}

In Fig. 1, survey spectra of the CdS covered standard and the KF-PDT treated CIGSe samples are shown for $6000 \mathrm{eV}$ excitation energy in the binding energy range from 0 to $2500 \mathrm{eV}$. Since the thickness of the CdS film is limited to a few nanometers, photoelectrons originating from the CIGSe absorber region still can be detected due to their increased kinetic energy which goes along with an increased information depth as compared to standard XPS. In addition, cadmium and sulfur related peaks appear in the spectra as expected. Moreover, small quantities of carbon and oxygen are traceable on both samples due to air contact after the absorber growth and due to the usage of aqueous solutions for the CBD of CdS. At first glance, both spectra look very similar, which emphasizes the high quality of the sample preparation. The fact that both, deposit (CdS) and substrate (CIGSe) peaks are visible in the spectra, allows the investigation of the CdS/CIGSe interface with depth resolving HAXPES.

Fig. 2 shows top view images of the standard CIGSe/CdS (a) and KF-PDT CIGSe/CdS (b) sample obtained with SEM. The morphology of both samples appears very similar with homogenous crystalline structures up to a size of $500 \mathrm{~nm}$, which can be related to different grains. The topmost CdS layer is not visible since the layer thickness is too small to be resolved with SEM. However, it is assumed that the CdS layer covers homogeneously the CIGSe absorber independent from morphology and structure of the surface due to the nature of the wet chemical deposition process ${ }^{16}$ and due to the very small lattice misfit between CIGSe and CdS leading to an epitaxial structure. ${ }^{17}$ Thus, the surface in the images mainly represents that of the bare CIGSe absorber. On a closer inspection, small precipitations with 


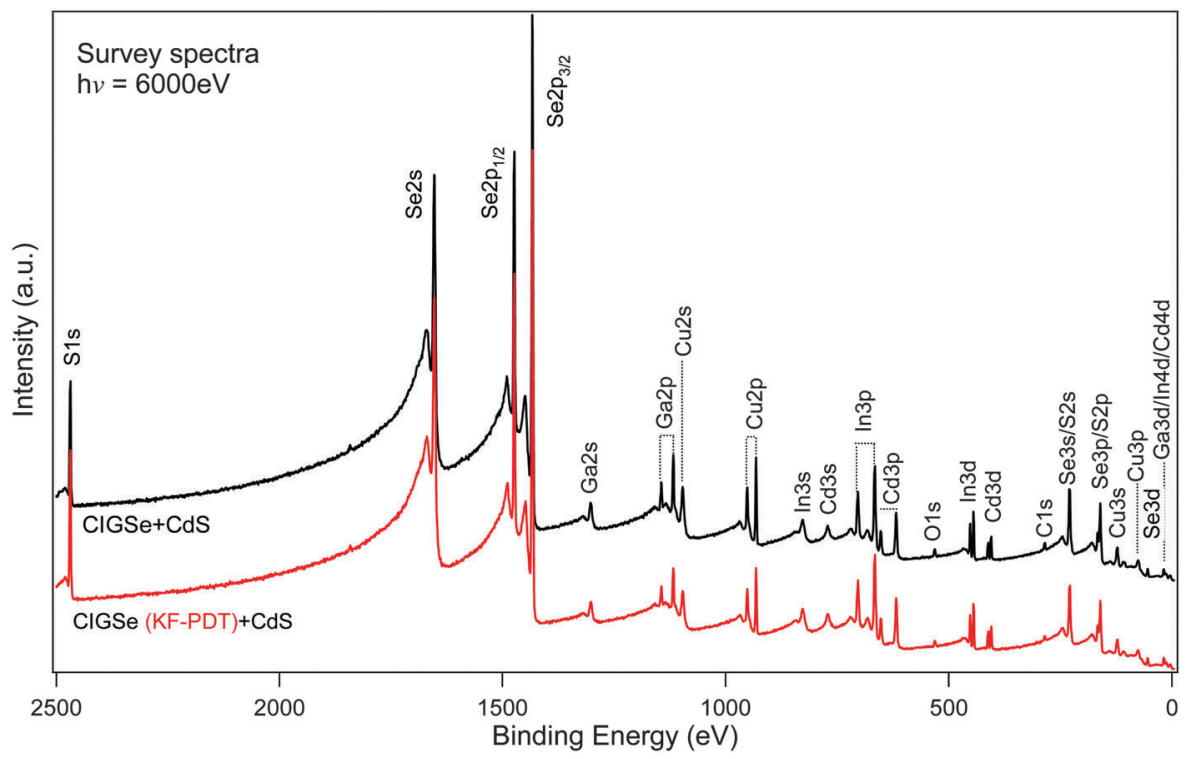

Fig. 1 XP survey spectra of the standard CIGSe/CdS (black) and KF-PDT CIGSe/CdS (red) sample measured at 6000 eV excitation energy.

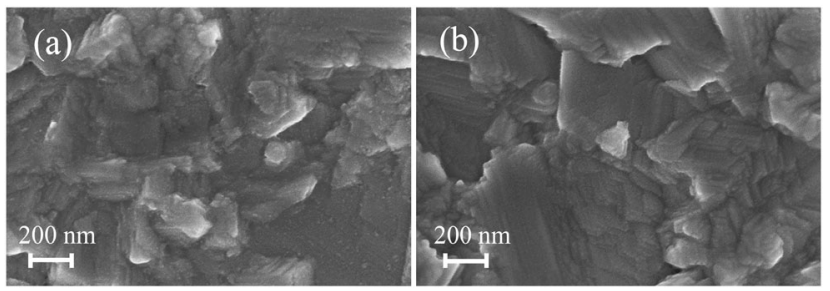

Fig. 2 SEM top view images of the standard CIGSe/CdS (a) and the KF-PDT CIGSe/CdS (b) sample which were also measured with HAXPES (see Fig. 1)

a diameter of less than $20 \mathrm{~nm}$ can be observed on the standard CIGSe/CdS sample (see Fig. 2(a)), which consist of $\mathrm{Cd}(\mathrm{OH})_{2}$ according to. ${ }^{18}$ In contrast, these entities are not visible on the KF-PDT CIGSe/CdS sample (see Fig. 2(b)). It seems that this phenomenon is related to the composition change at the CIGSe surface due to the KF treatment, which leads to a lower near surface $\mathrm{Cu}$ concentration as well as a higher near surface $\mathrm{Cd}$ and $\mathrm{S}$ concentration as shown later. In the following, similar aspects are discussed on an electronic level based on the HAXPES spectra.

Fig. 3 shows a compilation of normalized intensities derived from the Cu2 $\mathrm{p}_{3 / 2}, \operatorname{In} 3 \mathrm{p}_{3 / 2}, G a 2 \mathrm{p}_{3 / 2}, \operatorname{Se} 2 \mathrm{p}_{3 / 2}, \operatorname{Cd} 3 \mathrm{~d}_{5 / 2}$, and $\mathrm{S} 1 \mathrm{~s}$ peaks of the standard and the KF-PDT CIGSe/CdS sample. The shown values are calculated from the corresponding photoemission lines, which were measured with HAXPES at different excitation energies. Before starting the evaluation, all spectra were corrected with respect to their binding energy according to the position of the $\mathrm{Au}_{4 \mathrm{f}} \mathrm{f}_{7 / 2}$ peak located at $84.0 \mathrm{eV} \cdot{ }^{19}$ After subtraction of a Shirley background, the spectra were integrated in order to determine the respective line intensities. Then, they were normalized to the corresponding inelastic mean free path $\lambda$ (calculated by the TPP-2M formula ${ }^{20}$ using the QUASES $\operatorname{code}^{21}$ ), the transmission function of the analyzer $T$, and the partial subshell photoionization cross-section $\mathrm{d} \sigma / \mathrm{d} \Omega$ as tabulated in ref. 22 and 23. The parameters $\lambda, T$, and $\mathrm{d} \sigma / \mathrm{d} \Omega$ depend on the kinetic energy and therefore they vary for different excitation energies. The normalization procedure takes this dependency into account, thus allowing the direct comparison of normalized intensities of one species measured with different excitation energy. Additionally, the top axis of each graph shows the information depth (ID) for the corresponding core level signals which correlates to the excitation energy and which is estimated using the formula ID $\approx 3 \lambda .{ }^{19}$ The values vary from 4 to $24 \mathrm{~nm}$.

In the considered information depth regime, the normalized core level line intensities of the In and Se species are almost identical for both, the standard (black) and the KF-PDT (red) CIGSe/CdS samples, whereas the line intensities of the $\mathrm{Cu}$ and Ga species clearly differ from each other with increased values of the standard (black) CIGSe/CdS sample compared to the KF-PDT (red) CIGSe/CdS sample. In addition, the buffer layer species Cd and $\mathrm{S}$ of both samples are compared in Fig. 3(e) and (f), respectively. Within the given information depth, the KF-PDT CIGSe/CdS sample shows a higher line intensity for both, the $\mathrm{Cd}$ and $\mathrm{S}$ species with the latter one being more significant. In a first approximation this can be interpreted in terms of diffusion with $\mathrm{Cu}$ and $\mathrm{Ga}$ being repelled from the CIGSe/CdS interface and pushed further into the CIGSe bulk material while $\mathrm{Cd}$ and $\mathrm{S}$ are penetrating into the CIGSe interface region.

In Fig. 4, another presentation of the same issue is given by the ratio $I_{\mathrm{CIGSe}(\mathrm{KF}-\mathrm{PDT})+\mathrm{CdS}} / I_{\mathrm{CIGSe}+\mathrm{Cds}}$ over the excitation energy for all involved species. The shown values are referred to the Se related signal, which by definition is set to one, assuming that the KF-PDT does not affect the Se concentration at the CIGSe surface as demonstrated in Fig. 3(d). The advantage of this approach is to avoid the dependency on $\lambda, T$, and $\mathrm{d} \sigma / \mathrm{d} \Omega$ due to cancelling which otherwise might falsify the evaluation due to wrong assumptions or systematical errors. In the energy range from 2000 to $6000 \mathrm{eV}$ the $\mathrm{Cu} 2 \mathrm{p}$ signal steadily rises from 0.6 to 0.8 without crossing the reference line at one. This means that 

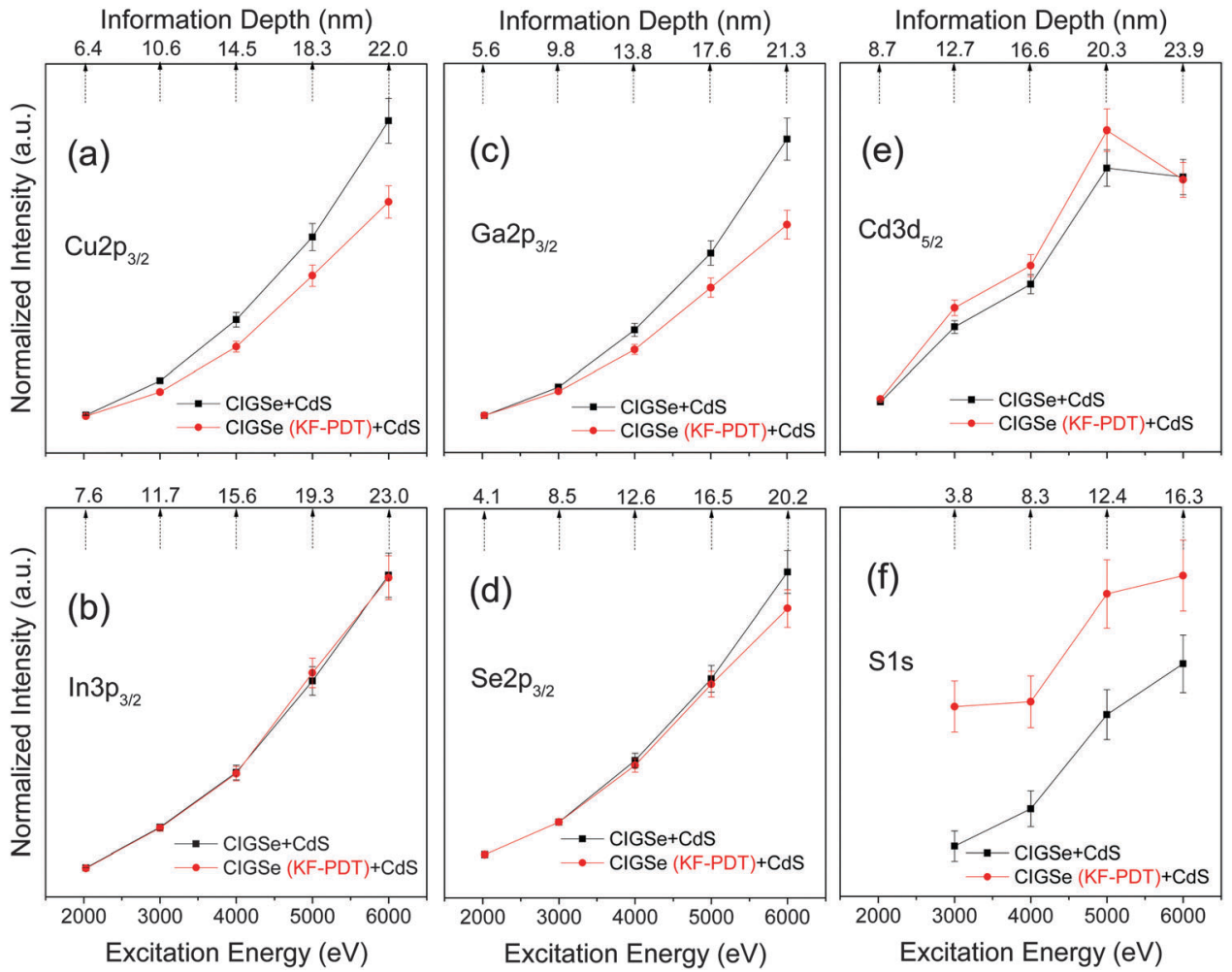

Fig. 3 Normalized intensities (the normalisation procedure is explained in the text) of absorber and buffer components as a function of excitation energy for the standard (black) and the KF-PDT (red) samples. The top axis shows the according information depth. For $2030 \mathrm{eV}$, the normalized intensity values are missing for the S1s related core levels since they cannot be excited with this energy.

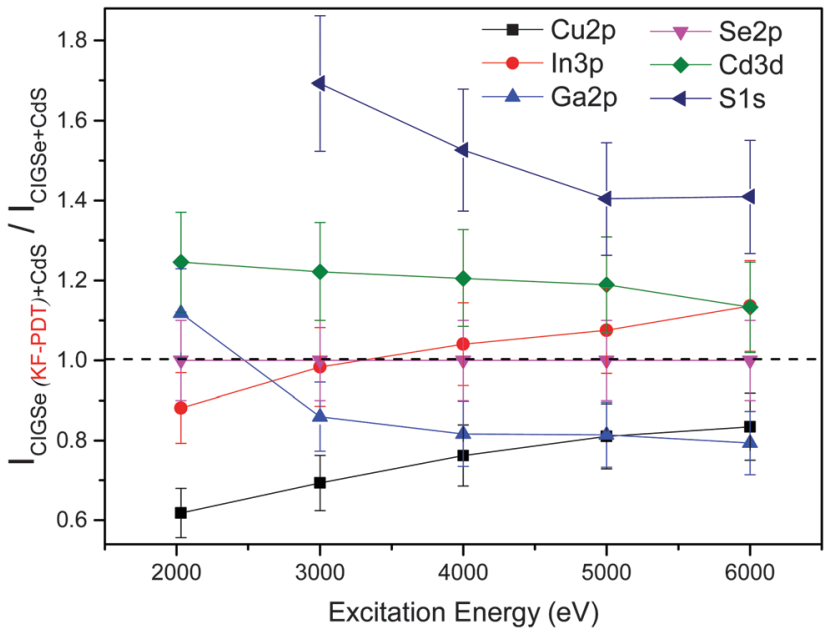

Fig. 4 Ratio of different core level intensities of the KF-PDT and the standard $\mathrm{CIGSe/CdS} \mathrm{samples.} \mathrm{All} \mathrm{values} \mathrm{are} \mathrm{referred} \mathrm{to} \mathrm{the} \mathrm{Se} \mathrm{related} \mathrm{signal} \mathrm{which} \mathrm{by}$ definition is set to one for each excitation energy value.

the $\mathrm{Cu}$ concentration in the KF-PD treated sample is always lower than in the standard sample. The fact that the Cu2p signal is not constant over the given energy range even implies that the $\mathrm{Cu}$ deficiency is more pronounced in the topmost region of the CIGSe material. On the other hand, the Cd3d signal behaves conversely and decreases steadily from 1.3 to 1.1 within the same energy range, which means that there is more
Cd on the KF-PD treated CIGSe surface than on the standard one. Moreover, within an assumed error of $\pm 10 \%$, the In $3 p$ value appears more or less constant and close to 1 , which points to an equal near-surface distribution of In in both samples, similar to the Se case. However, a slight tendency for an In enrichment for higher excitation energies and thus deeper regions cannot be denied. A similar behaviour but with an opposite effect can be found for the Ga2p signal. There, an initial Ga excess turns into a significant depletion for higher energies. We attribute this to specific interface reactions during the CIGSe/CdS heterojunction formation. Nevertheless, the most pronounced difference is observed for the S1s signal, which steadily goes from 1.7 to 1.5 within the given energy range of 3000 to $6000 \mathrm{eV}$. This means that on the KF-PD treated CIGSe sample the amount of $\mathrm{S}$ is much higher than on the standard sample. This can be only explained by unknown effects during the CdS formation which might be influenced by the $\mathrm{Cu}$ and $\mathrm{Ga}$ depletion. We will discuss this point later on together with other findings.

Fig. 5 compares the $[\mathrm{Cu}] /([\mathrm{In}]+[\mathrm{Ga}])(\mathrm{CGI})$, the $[\mathrm{Ga}] /([\mathrm{In}]+[\mathrm{Ga}])$ (GGI), and the $[\mathrm{Cd}] /[\mathrm{Cu}]$ ratios as a function of excitation energy for both samples under investigation based on normalized intensities.

In case of the standard CIGSe/CdS sample, the CGI (see Fig. 5(a)) varies only slightly between 0.82 and 0.88 , which stands for a homogeneous $\mathrm{Cu} / \mathrm{III}$ distribution over the probed depth. On the other hand the GGI (see Fig. 5(b)) rises strongly from 0.29 to 0.40 below $4000 \mathrm{eV}$ before it stabilizes. In contrast, this behaviour is 

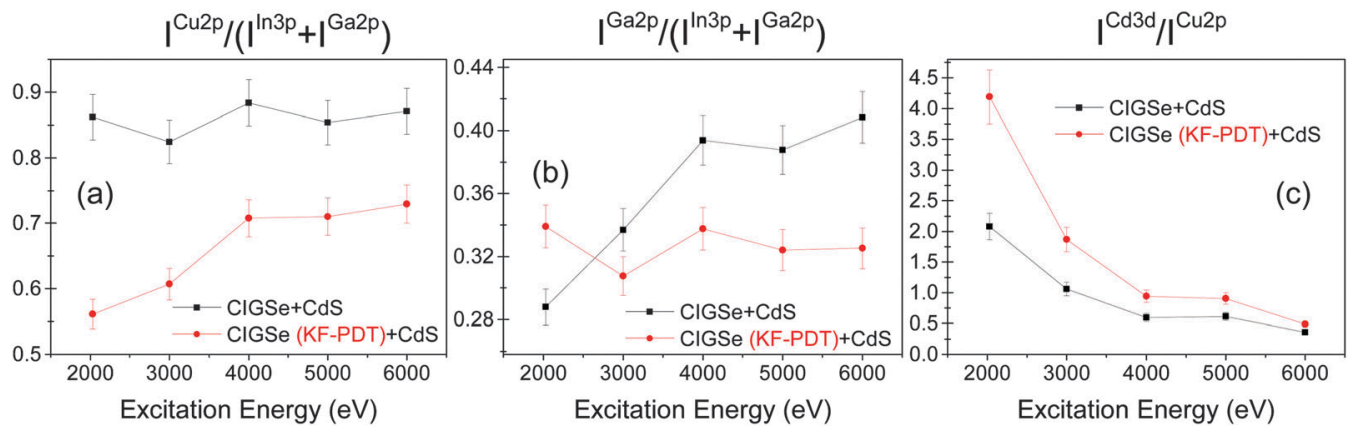

Fig. 5 Selected concentration ratios as derived from normalized intensities. (a) [Cu]/([Ga] + [In]) (CGI), (b) [Ga]/([Ga] + [In]) (GGl), and (c) [Cd]/[Cu] as a function of excitation energy.

completely different for the KF-PDT CIGSe/CdS sample. The CGI starts at a relatively low value of 0.55 before it stabilizes at 0.71 whereas the GGI remains almost constant with a value of 0.32 over the considered sample depth range. On closer examination it seems that there is a correlation between both parameters (CGI and GGI). While for the standard CIGSe/CdS sample the GGI rises and the CGI is constant with increasing depth the same parameters behave in opposite way for the KF-PDT CIGSe/CdS sample on a lower level. On both samples, the CGI affects the GGI and vice versa. Considering the shape of the $\mathrm{Cd} / \mathrm{Cu}$ ratio, this is similar for both samples. In the lower excitation energy range it starts at 4.2 and 2.1 for the KF-PDT and the standard CIGSe/CdS sample, respectively. For higher excitation energies it approaches zero which is reasonable since the diffusion of Cd into the CIGSe absorber is depth limited.
In Fig. 6, various spectra of the near valence band region at different excitation energies are shown. The spectra are calibrated according to the gold reference and they are normalized to the $\operatorname{In} 4 \mathrm{~d}_{5 / 2}$ peak intensity for a better comparison. With increasing photon energy, which is equivalent to an increasing information depth, a relative decrease of the Cd4d signal compared to the In4d signal is observed. In general, the height of the Cd4d peak of the KF-PDT sample (red curve) exceeds that of the standard sample (black curve) but the difference decreases with increasing excitation energy. This is in accordance to the behaviour shown in Fig. 4. In addition, a distinct shift of the core levels and the valence band edges of the KF-PDT sample towards lower binding energies compared to the standard sample is visible. The resolution of HAXPES is sufficient to assign the valence band maximum with respect to the Fermi level for different

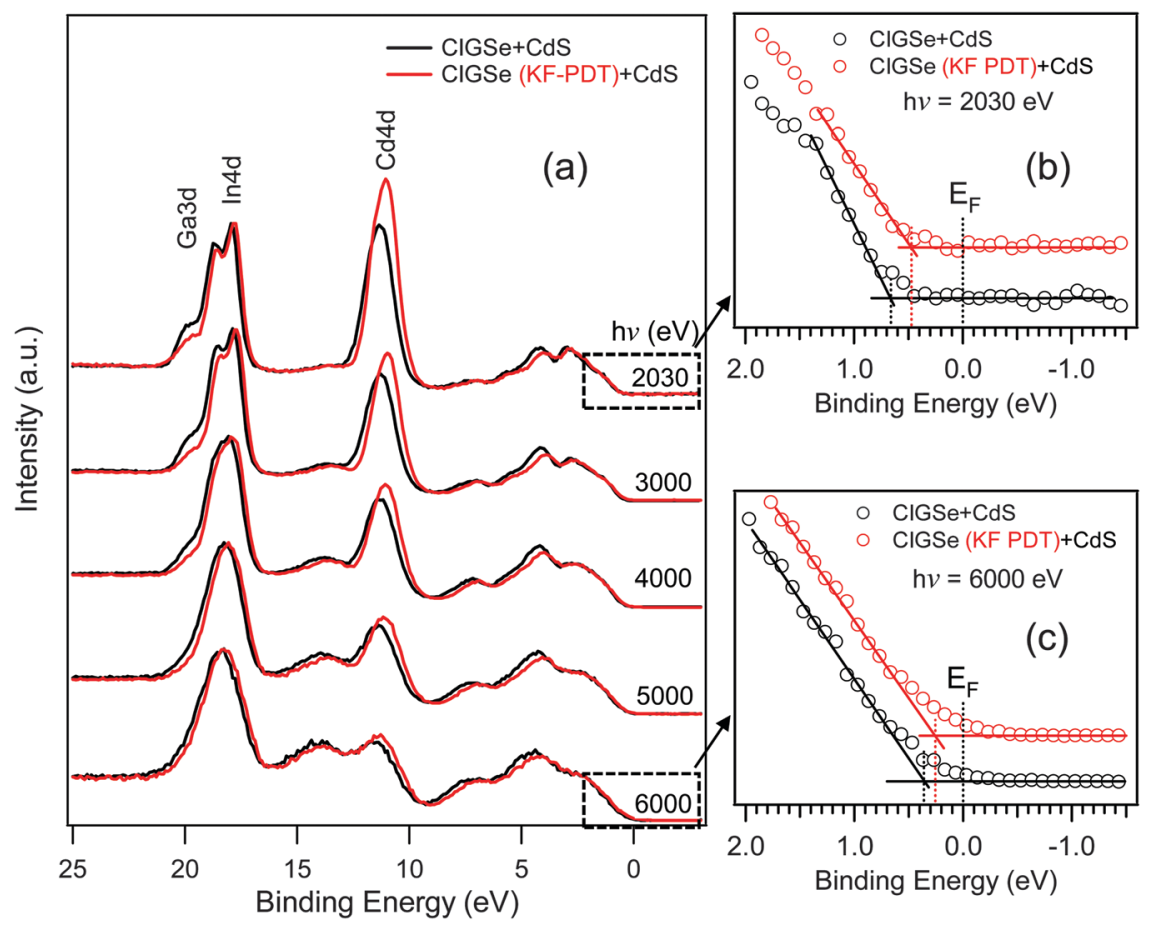

Fig. 6 (a) XP spectra of the near valence band region of the standard CIGSe/CdS (black) and KF-PDT CIGSe/CdS (red) sample measured for various excitation energies. All spectra are normalized to the $\ln 4 d_{5 / 2}$ signal. ( $b$ and $c$ ) Onsets of the upper valence band of two examples with drawn in linear extrapolations to demonstrate how the $E_{\mathrm{VBM}}-E_{\mathrm{F}}$ values are determined, which are visualized in Fig. 7. 


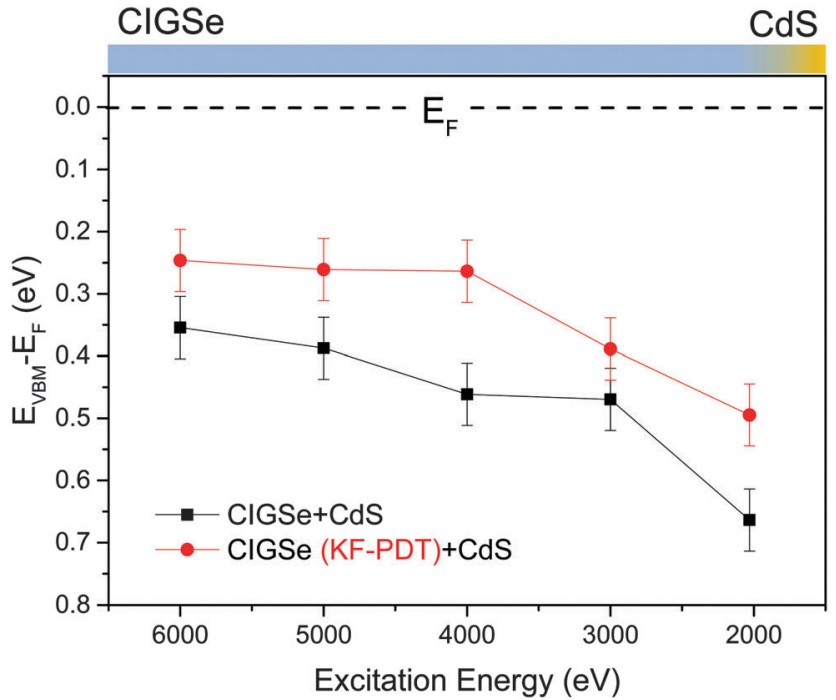

Fig. 7 The position of the valence band maxima with respect to the Fermi level measured at different excitation energies providing depth dependent information with an estimated uncertainty of \pm 0.05 .

excitation energies. It was determined by linear extrapolation of the valence band onset with respect to the background level assuming a maximum error of $\pm 0.05 \mathrm{eV}$ which strongly depends on the shape of the valence band edge. Two examples for the determination of the valence band maximum with respect to Fermi level are given in Fig. 6(b) and (c) for the excitation energies of $2030 \mathrm{eV}$ and $6000 \mathrm{eV}$, respectively. The results are shown in Fig. 7 with a schematic representation of the CIGSe/CdS heterocontact on the top of the graph that correlates with the detection depth using different excitation energies.

The dashed line in the graph represents the energetic position of the Fermi level. However, it is important to know that the measured valence band offset belongs to CIGSe although this material is covered by CdS. This is due to the fact that the band gap of CIGSe is much smaller than the band gap of CdS thus hiding it. Furthermore, the amount of CdS is small compared to CIGSe which leads to a weaker valence band signature. In general, the binding energy in the valence band region is low resulting in a comparatively large kinetic energy of the considered photoelectrons originating from this region. This means that the information depth is even more increased which further explains the dominance of the CIGSe valence band in the spectra. As can be clearly seen in the graph there is a distinct shift of the valence band maximum to lower values for the KF-PDT sample of about $0.15 \mathrm{eV}$. In addition, a pronounced band bending downwards in the near-surface region is observed on both samples with a shift of $0.3 \mathrm{eV}$ between the first and the last data point at $2030 \mathrm{eV}$ and $6000 \mathrm{eV}$, respectively.

In Fig. 8, a compilation of the binding energy positions of selected core level peaks is shown with respect to the excitation energy. All values are referred to the Fermi level, thus the graphs can be directly compared to the valence band maximum shown in Fig. 7. Except for the value of the $\mathrm{Ga}_{2} \mathrm{p}_{3 / 2}$ peak in the near-surface region at $2030 \mathrm{eV}$, there is a more or less constant shift of about $0.2 \mathrm{eV}$ of the KF treated CIGSe sample towards lower binding energies compared to the untreated sample. In contrast to the course of the valence band over the excitation energy, the binding energy values of the core levels show a bowlike behaviour. The $\mathrm{Cu}, \mathrm{In}, \mathrm{Ga}$, and $\mathrm{Cd}$ peaks initially follow the course of the valence band with increasing thickness towards higher binding energies, whereas the "anionic" species S and Se stay on a constant level. However, the first binding energy value of the S1s peak is unknown since the S1s transition is not accessible at $2030 \mathrm{eV}$. With increasing excitation energy the CL peaks shift again to higher binding energies forming the above mentioned bow. The fact that the valence band and also the core levels are not shifting equally with excitation energy cannot be explained by band bending due to doping. It is important to emphasize that the shape of the curves (CL peaks and valence band maximum) and the constant shift between both samples are due to different effects.

\section{Discussion}

The fact that the measured valence band offset of the KF treated CIGSe/CdS sample is smaller compared to that of the untreated one first causes some irritations, since the opposite behaviour is expected according to ref. 24 . The deviation can only be explained by the presence of a thin CdS layer affecting the interface properties of the CIGSe/CdS contact. The valence band maximum of the untreated CIGS/CdS sample in the near interface region recorded at $2030 \mathrm{eV}$ is $0.7 \pm 0.05 \mathrm{eV}$, which is in the range of previously reported values ${ }^{25}$ for the same material system. This agreement gives us a positive assessment on the reliability of our approach including the measurement of the KF treated sample showing its valence band maximum at $0.55 \pm 0.05 \mathrm{eV}$, which is roughly $0.15 \mathrm{eV}$ above that of the untreated sample. Moreover, the observed behaviour is independent from the excitation energy with a more or less constant offset of $0.15 \mathrm{eV}$, which follows the course of the valence band with increasing information depth. A similar shift is also observed for the CL peaks of all involved species except for gallium and sulphur in the near interface region. There is obviously a consistent shift of all the spectral features of the KF treated CIGSe sample to lower binding energies due to the incorporation of a negative charge into the interface region or due to the formation of an interfacial dipole (see Fig. 9(a)). For simplification, the binding energy positions of the CL peaks are represented as straight lines. Furthermore, in the near surface regime the binding energy of the Ga2 $\mathrm{p}_{3 / 2}$ peak is higher and the one of the S1s peak is lower than expected compared to the untreated sample (see Fig. 8(c) and (f)). This finding goes along with increased intensities for both species as can be seen in Fig. 4 for an excitation of 2030 eV. Possibly, an additional, intermediate, and ultrathin $\mathrm{Ga}_{x} \mathrm{~S}_{y}$ phase was formed on the KF treated CIGSe sample during the deposition of CdS, which now affects the band alignment. In addition, the interface of the KF treated sample seems to be changed (see Fig. 2) so that binding states of interlinked species forming the heterojunction become visible. ${ }^{26}$ However, due to charge neutrality reasons it is difficult 

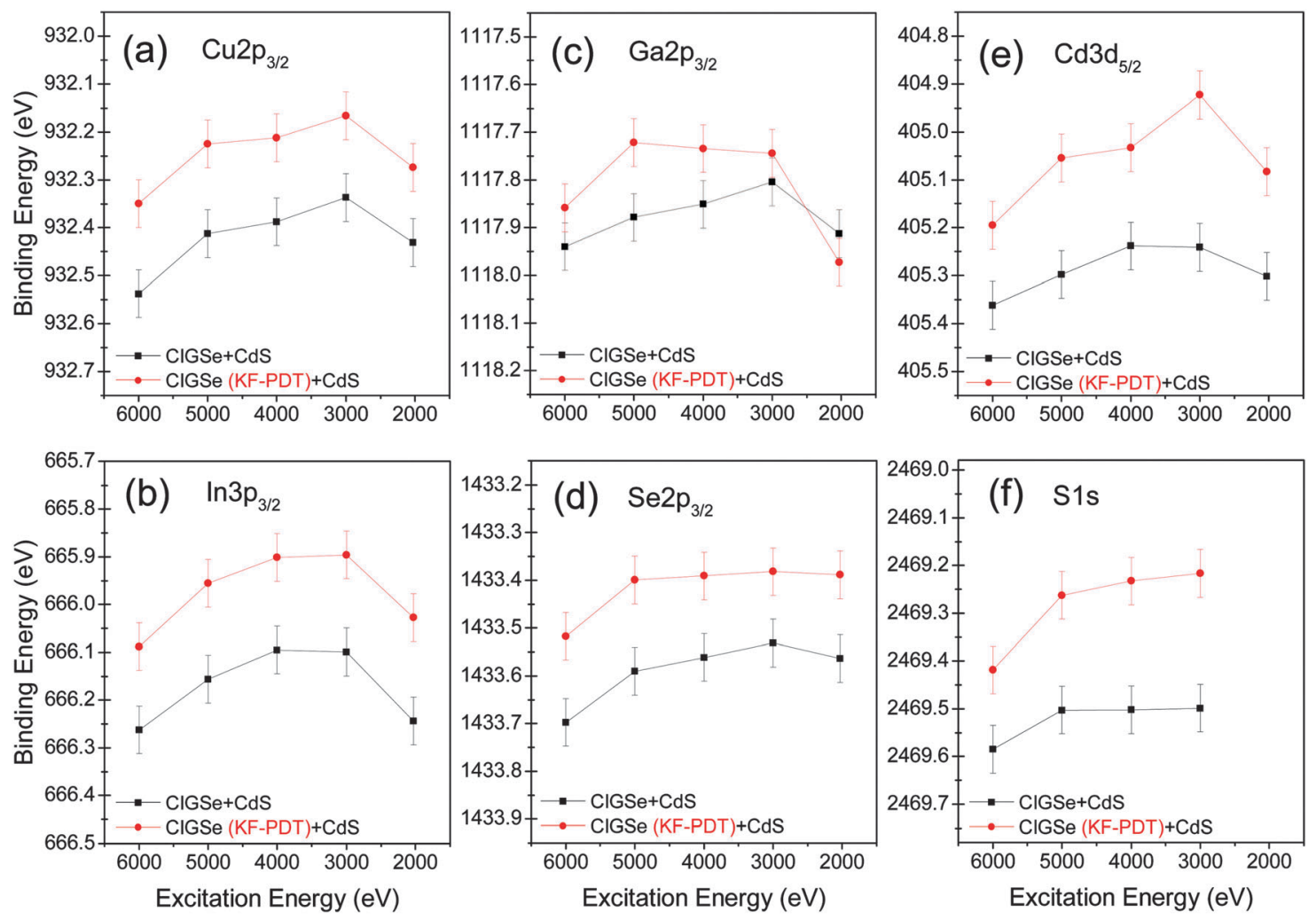

Fig. 8 Binding energy positions of selected core level (CL) peaks as a function of excitation energy (a-f).
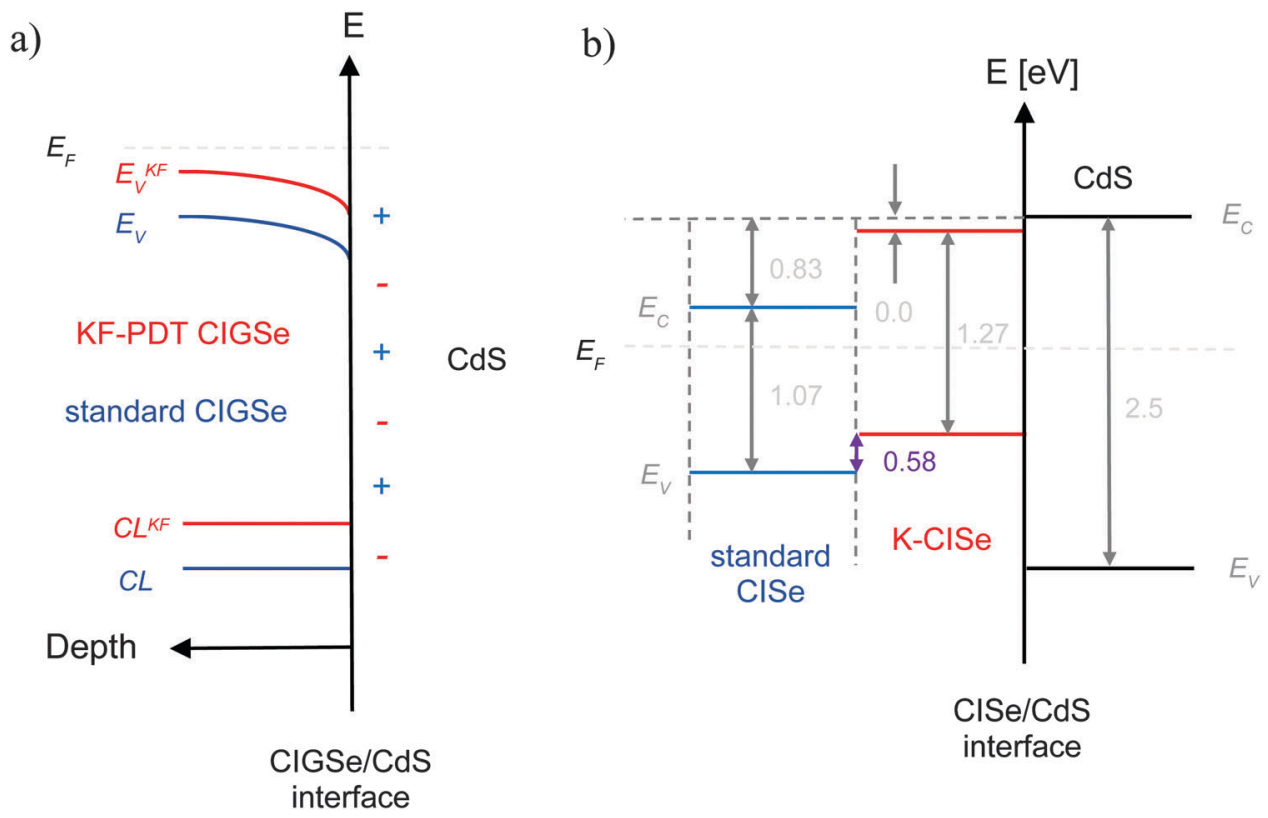

$$
\begin{aligned}
& \mathrm{CISe} / \mathrm{CdS} \\
& \text { interface }
\end{aligned}
$$

Fig. 9 (a) Scheme of the CIGSe/CdS interface showing the relative positions of the valence band and the CL peaks for the standard (blue) and the KF-treated (red) CIGSe samples. (b) Theoretical band alignment of $\mathrm{Cu}$-poor $\mathrm{CISe}\left(\mathrm{CulnSe}_{2}\right)$ and $\mathrm{Cu}$-poor $\mathrm{K}$ containing CISe with respect to $\mathrm{CdS}$ according to ref. 27.

to explain that CdS related peaks also shift into the same direction on the KF treated sample. Hence, other reasons than the formation of an interface dipole must be responsible for this shift which still is related to electrostatic fields induced by additional charge.
In Fig. 9(b), a theoretical scheme of the band alignment of $\mathrm{Cu}$-poor CISe (blue) and Cu-poor K containing CISe (red) with respect to CdS is shown according to ref. 27. The calculation used hybrid DFT functionals (B3PW91, ${ }^{28}$ ) yielding good estimates for chalcopyrite band gaps. As basis, $\mathrm{Cu}_{5} \mathrm{In}_{9} \mathrm{Se}_{16} / \mathrm{CdS}(110)$ and 
$\mathrm{K}_{2} \mathrm{Cu}_{3} \mathrm{In}_{9} \mathrm{Se}_{16} / \mathrm{CdS}(110)$ super cells are used to determine the band discontinuities of the Cu-poor CISe/CdS and K-CISe/CdS heterocontacts. By applying the transitivity rule, ${ }^{29}$ it turns out that the valence band maximum of the potassium containing, copper poor CISe absorber is located 0.58 above the standard CISe absorber in contact to CdS and this is what we see by trend in our measurements. Although the theoretical work is only related to CISe and not to CIGSe, the obtained results demonstrate that it is possible to have a lower valence band maximum at the interface due to the incorporation of $\mathrm{K}$. However, the increased amount of $\mathrm{S}$ causes a larger absorber band gap at the interface, pointing to a decreased conduction band discontinuity when considering the pure CIGSe absorber in contact to the CdS buffer. Unfortunately, the conduction band is not accessible within the given experimental setup so that this phenomenon cannot be proven further.

Another point is the development of the CGI and GGI ratios on both samples with increasing thickness (Fig. 5). On the KF treated CIGSe absorber the overall concentration of $\mathrm{Cu}$ is smaller than on the non-treated absorber. Additionally, there is a gradient in the near-surface regime pointing to an enhanced $\mathrm{Cu}$-depletion. In contrast to this, the standard CIGSe absorber is less Cu-poor and the CGI ratio shows a constant behaviour over the whole thickness range within the error limit. So far it is assumed that the bare CIGSe surface is stronger Cu-depleted at the very-near surface than in the bulk which might be attributed to the occurrence of an ordered defect compound (ODC) phase ${ }^{30}$ or to the reconstruction of the topmost CIGSe layer [10]. Following the results of our measurements, this can be only confirmed for the KF treated sample. Possibly, this effect correlates with the GGI ratio, which shows an opposite behaviour. While for the KF treated absorber the GGI ratio is nearly constant $(\sim 0.32)$ over the complete energy range, it starts from a lower value for the standard sample, steadily increasing and ending up at $\sim 0.4$. The near-surface depletion of $\mathrm{Cu}$ or $\mathrm{Ga}$ appears either on the KF-treated or on the non KF-treated CIGSe absorber, respectively. In addition, the Cd concentration is much higher for the KF-treated sample compared to standard one. Obviously, Cd diffuses much easier into the KF-treated CIGSe absorber. This goes along with an increased $\mathrm{S}$ concentration over the complete thickness range (Fig. 4) which could be explained by a stronger interdiffusion at the interface of the KF-treated CIGSe sample or a higher sulphur concentration in the CdS buffer layer at all. Besides this argumentation it is also possible that the total amount of CdS is higher on the KF-treated sample because the growth conditions for CdS are apparently improved. Moreover, it seems that the KF-PDT has an additional impact on the heterocontact formation by changing the pristine CIGSe surface and its concentration ratios accordingly, e.g. by a higher Ga or S content in the near surface regime of the CIGSe absorber, which influences the band gap.

In parallel, a strong $\mathrm{Cu}$ depletion at the surface should lead to a band gap widening by lowering the onset of the upper valence band due to the missing repulsing effect of the Cu3d valence states. ${ }^{31}$ This behaviour can be directly observed in Fig. 7 with a pronounced course of the valence band maximum over the excitation energy in both cases, the KF-treated as well as the non KF-treated CIGSe absorber, respectively. We attribute this band bending downwards to a decrescent $\mathrm{Cu}$ concentration towards the surface and not to the formation of a space charge region due to doping which would go several $100 \mathrm{~nm}$ into the depth. Since the variation of the valence band maximum is strongest in the excitation energy range between 2000 to $4000 \mathrm{eV}$, we assume that the $\mathrm{Cu}$ depletion at the interface is limited to a thickness of 2-5 nm only. In this thickness regime the change of the onset of the upper valence band is mostly pronounced.

In addition to the enhanced $\mathrm{Cu}$ depletion on the $\mathrm{KF}$ treated CIGSe sample, which was reported earlier ${ }^{7}$ and which was also found in this work as shown in Fig. 4 and 5, we observe an enrichment of $\mathrm{Cd}$ in the interface region. We ascribe this to the fact that Cd diffuses into the CIGSe absorber and occupies the $\mathrm{Cu}$ vacancies as published before. ${ }^{11}$ This finding is also supported by the deviation of the concentration levels of these two species which are of the same order of magnitude in both samples (see Fig. 4). In other words, the lack of $\mathrm{Cu}$ in the $\mathrm{KF}$ treated sample is almost fully compensated by the presence of $\mathrm{Cd}$. But what is the impact of the surplus of Cd atoms in CIGSe? We assume, that the optimum CIGSe absorber surface for high efficient solar cells is Cu-poor and already inverted, thus changing from p-type conduction in the bulk to n-type conduction at the surface. ${ }^{32}$ Therefore, additional Cd, which acts as donor in $\mathrm{CIGSe}^{33}$ can release further electrons into the conduction band as free charge carriers. These electrons then migrate as majority carriers along the conduction band towards the interface. If now the conduction band discontinuity between the CIGSe absorber and the CdS buffer layer as a barrier is not too large, they are able to overcome this barrier and accumulate at the CdS surface as negative charges. The amount of this charge is directly correlated to the $\mathrm{Cd}$ concentration in the CIGSe absorber. The given explanation reflects exactly what we have measured. In addition, we conclude that the barrier in the conduction band at the heterocontact is small enough otherwise the accumulation would take place in the interface region. With an extrapolated value of the valence band discontinuity between CIGSe and CdS of about $0.9 \mathrm{eV},{ }^{34,35}$ a valence band offset of $0.5 \mathrm{eV}$ at the KF treated CIGSe surface as measured and shown in Fig. 7, and a band gap of the CdS buffer layer of $2.5 \mathrm{eV},{ }^{36}$ a widening of the CIGSe band gap up to a value of $1.6 \mathrm{eV}$ at the interface due to the incorporation of $\mathrm{S}$ and $\mathrm{Ga}$ leads to a negligible conduction band discontinuity. A smaller value than $1.6 \mathrm{eV}$ as well as a larger one would imply either a band bending downwards or upwards on the chalcopyrite side of the junction which for a fixed conduction band position of the CdS layer with respect to the Fermi level would lead in both cases to a potential barrier affecting the electron transfer through the interface. However, further simulations are necessary to consolidate this assumption, but the experimental data strongly point into this direction.

Besides the ongoing discussion, there is another aspect which is worth to be mentioned and this is the morphology of the CdS layer. In contrast to the KF treated CIGSe sample, there are small precipitations visible on the standard sample (Fig. 2) directing to a slightly different growth mechanism when applying the 
CBD and, which hints to a less ordered interface or to an increased lattice misfit. The lattice parameter of CIGSe $(a=0.56-0.58 \mathrm{~nm}$ depending on the Ga content); is in the range of that of CdS $(a=0.58 \mathrm{~nm}) .{ }^{37}$ This means that stress induced defect formation in the initial growth of the CdS due to lattice mismatch is unlikely. Obviously, the standard CIGSe absorber owns more surface defects, which are acting as nucleation centers during the subsequent CdS deposition forming additional precipitations. Further investigations concerning this aspect are necessary.

\section{Conclusion}

We have investigated the impact of the KF-PDT on CIGSe by means of depth resolved hard X-ray photoemission spectroscopy in the near surface region. Two similar absorbers are used with one being treated by potassium fluoride prior to the chemical bath deposition of an ultrathin CdS layer while the other was not. During this study we have tried to keep all parameters the same except the KF-PDT in order to carve out the impact of this treatment. It turns out that besides strong chemical changes in the CIGSe/CdS interface region, namely a $\mathrm{Cu}$ depletion which goes along with a $\mathrm{Cd}$ accumulation, the electronic properties of the heterocontact are significantly affected and influenced by the KF-PDT. We attribute this to an enhanced doping effect, which is induced by additional $\mathrm{Cd}$ in the near surface region and, which releases free electrons into the conduction band of the apparently inverted CIGSe absorber with electrons regarded then as majority carriers. These electrons are able to follow the band downwards to the surface where they accumulate. It is assumed that this process starts already during the deposition of CdS and that excessive electrons are able to overcome the building-up potential barrier at the CIGSe/CdS interface. By this effect the band alignment of the CIGSe/CdS heterojunction is obviously improved, leading to better efficiencies of the CIGSe based thin film solar cell devices. Furthermore, we found out that the CIGSe/CdS interface is strongly intermixed and that the course of the valence band in the very near surface region of CIGSe of both samples is basically of chemical nature and not only due to band bending. However, on the KF treated sample we have observed a significant increase of the sulfur concentration compared to the untreated sample. We assume that this phenomenon leads to a dedicated band gap widening which mainly affects the position of the conduction band in such a way that the height of the barrier at the interface is reduced. However, since we are not able to probe the conduction band with our experimental approach this predication remains speculative and needs further experimental proof.

\section{Acknowledgements}

The authors thank J. Kavallakat for the SEM images and the Bessy staff for their excellent support. B. Ümsür gratefully acknowledges financial support from the Turkish Scholarship Council of Ministry of Education of the Republic of Turkey (MEB). Moreover, the support of the German federal environment ministry (BMU) in the framework of the ComCIGS2 project (grant number 0324448D) is also thankfully acknowledged.

\section{References}

1 P. Jackson, D. Hariskos, R. Wuerz, O. Kiowski, A. Bauer, T. M. Friedlmeier and M. Powalla, Phys. Status Solidi RRL, 2015, 9, 28-31.

2 R. Caballero, C. A. Kaufmann, T. Eisenbarth, M. Cancela, R. Hesse, T. Unold, A. Eicke, R. Klenk and H. W. Schock, Thin Solid Films, 2009, 517, 2187-2190.

3 R. Caballero, C. A. Kaufmann, T. Eisenbarth, A. Grimm, I. Lauermann, T. Unold, R. Klenk and H. W. Schock, Appl. Phys. Lett., 2010, 96, 092104.

4 R. Wuerz, A. Eicke, F. Kessler, P. Rogin and O. Yazdani-Assl, Thin Solid Films, 2011, 519, 7268-7271.

5 L. E. Oikkonen, M. G. Ganchenkova, A. P. Seitsonen and R. M. Nieminen, J. Appl. Phys., 2013, 114, 083503.

6 S. Puttnins, S. Levcenco, K. Schwarzburg, G. Benndorf, F. Daume, A. Rahm, A. Braun, M. Grundmann and T. Unold, Sol. Energy Mater. Sol. Cells, 2013, 119, 281-286.

7 A. Chirilă, P. Reinhard, F. Pianezzi, P. Bloesch, A. R. Uhl, C. Fella, L. Kranz, D. Keller, C. Gretener, H. Hagendorfer, D. Jaeger, R. Erni, S. Nishiwaki, S. Buecheler and A. N. Tiwari, Nat. Mater., 2013, 12, 1107-1111.

8 J. Kiss, T. Gruhn, G. Roma and C. Felser, J. Phys. Chem. C, 2013, 117, 25933-25938.

9 J. Kiss, T. Gruhn, G. Roma and C. Felser, J. Phys. Chem. C, 2013, 117, 10892-10900.

10 H. Mönig, C. H. Fischer, A. Grimm, B. Johnson, C. A. Kaufmann, R. Caballero, I. Lauermann and M. C. Lux-Steiner, J. Appl. Phys., 2010, 107, 113540.

11 B. Ümsür, W. Calvet, B. Höpfner, A. Steigert, I. Lauermann, M. Gorgoi, K. Prietzel, H. A. Navirian, C. A. Kaufmann, T. Unold and M. C. Lux-steiner, Thin Solid Films, 2015, 582, 366-370.

12 C. A. Kaufmann, T. Unold, D. Abou-Ras, J. Bundesmann, A. Neisser, R. Klenk, R. Scheer, K. Sakurai and H.-W. Schock, Thin Solid Films, 2007, 515, 6217-6221.

13 C. A. Kaufmann, A. Neisser, R. Klenk and R. Scheer, Thin Solid Films, 2005, 480-481, 515-519.

14 M. Gorgoi, S. Svensson, F. Schäfers, G. Öhrwall, M. Mertin, P. Bressler, O. Karis, H. Siegbahn, A. Sandell, H. Rensmo, W. Doherty, C. Jung, W. Braun and W. Eberhardt, Nucl. Instrum. Methods Phys. Res., Sect. A, 2009, 601, 48-53.

15 F. Schaefers, M. Mertin and M. Gorgoi, Rev. Sci. Instrum., 2007, 78, 123102.

16 J. Han, C. Liao, L. Cha, T. Jiang, X. Hua-mu, Z. Kui and M.-P. Besland, J. Phys. Chem. Solids, 2014, 75, 1279-1283.

17 T. Nakada and A. Kunioka, Appl. Phys. Lett., 1999, 74, 2444.

18 T. M. Friedlmeier, D. Braunger, D. Hariskos, M. Kaiser, H. N. Wanka and H. W. Schock, in 25th IEEE Photovoltaic Specialists Conference, Washington, D. C., 1996, pp. 845-848. 
19 D. Briggs and M. P. Seah, Practical Surface Analysis, John Wiley \& Sons, Chichester, 1983.

20 S. Tanuma, C. J. Powell and D. R. Penn, Surf. Interface Anal., 1993, 21, 165-176.

21 S. Tougaard, QUASES-IMFP-TPP2M program, Quases-Tougaard Inc., 2002.

22 M. B. Trzhaskovskaya, V. I. Nefedov and V. G. Yarzhemsky, At. Data Nucl. Data Tables, 2001, 77, 97-159.

23 M. B. Trzhaskovskaya, V. I. Nefedov and V. G. Yarzhemsky, At. Data Nucl. Data Tables, 2002, 82, 257-311.

24 P. Pistor, D. Greiner, C. A. Kaufmann, S. Brunken, M. Gorgoi, A. Steigert, W. Calvet, I. Lauermann, R. Klenk, T. Unold and M.-C. Lux-Steiner, Appl. Phys. Lett., 2014, 105, 063901.

25 M. Morkel, L. Weinhardt, B. Lohmüller, C. Heske, E. Umbach, W. Riedl, S. Zweigart and F. Krag, Appl. Phys. Lett., 2001, 79, 4482-4484.

26 W. Calvet, H.-J. Lewerenz and C. Pettenkofer, Phys. Status Solidi A, 2014, 211, 1981-1990.

27 H. Xiao and W. A. Goddard, J. Chem. Phys., 2014, 141, 094701.

28 A. D. Becke, J. Chem. Phys., 1993, 98, 5648-5652.
29 N. E. Christensen, Phys. Rev. B: Condens. Matter Mater. Phys., 1988, 38, 12687-12690.

30 S. B. Zhang, S.-H. Wei, A. Zunger and H. Katayama-Yoshida, Phys. Rev. B: Condens. Matter Mater. Phys., 1998, 57, 9642-9656.

31 J. L. Shay, B. Tell, H. M. Kasper and L. M. Schiavone, Phys. Rev. B: Condens. Matter Mater. Phys., 1972, 5, 5003-5005.

32 A. Rockett, D. Liao, J. T. Heath, J. D. Cohen, Y. M. Strzhemechny, L. J. Brillson, K. Ramanathan and W. N. Shafarman, Thin Solid Films, 2003, 431-432, 301-306.

33 B. Johnson, J. Klaer, A. Vollmer and I. Lauermann, J. Vac. Sci. Technol., A, 2012, 30, 04D114.

34 T. Löher, W. Jaegermann and C. Pettenkofer, J. Appl. Phys., 1995, 77, 731.

35 A. Klein, J. Fritsche, W. Jaegermann, J. H. Schön, C. Kloc and E. Bucher, Appl. Surf. Sci., 2000, 166, 508-512.

36 J. I. Pankove, Optical Processes in Semiconductors, Dover Publications, Inc., New York, 1971.

37 D. Strauch, in New Data and Updates for several III-V (including mixed crystals) and II-VI Compounds, ed. U. Rössler, Springer, Berlin Heidelberg, 2012, pp. 99-102. 